\title{
DIE EINSTELLUNG DER DAF-LEHRENDEN ZUR GRAMMATIK IM DAF-UNTERRICHT AM BEISPIEL SLOWENISCHER ABITURPRÜFENDER
}

Jahrelang war die Fremdsprachendidaktik mit der Suche nach der besten Methode für den Fremdsprachenunterricht beschäftigt. Der Grammatik wurde dabei ein äußerst unterschiedlicher Stellenwert zugeschrieben. Denken wir etwa an die Grammatik-Übersetzungsmethode auf der einen und die audio-linguale Methode auf der anderen Seite. Die Existenz einer universell zu favorisierenden Methode zum Erlernen von Fremdsprachen ist allerdings bis heute nicht nachgewiesen worden. In der heutigen Postmethodenära des Fremdsprachenunterrichts spricht man eher über didaktisch-methodische Prinzipien, auf denen der Sprachunterricht beruhen soll.

Als das wichtigste im Fremdsprachenunterricht zu erreichende Lernziel wird im Allgemeinen die kommunikative Kompetenz verstanden, die aus einer linguistischen, einer soziolinguistischen und einer pragmatischen Komponente besteht. Der Europarat bezeichnet in seinem Dokument „Gemeinsamer europäischer Referenzrahmen für Sprachen: Lernen, lehren, beurteilen“ (weiter GeR) (2001) die grammatische Kompetenz, worunter die Fähigkeit, Sätze zur Vermittlung von Bedeutungen zu strukturieren, verstanden wird, allerdings als zentralen Bestandteil der kommunikativen Kompetenz.

Dieser Beitrag setzt sich zweierlei zur Aufgabe: erstens, den Stellenwert von Grammatik im Fremdsprachenunterricht der Postmethodenära kurz zu skizzieren, und zweitens, am Beispiel externer Bewerter und Bewerterinnen der slowenischen Abiturprüfung im Fach Deutsch als einer spezifischen Gruppe von DaF-Lehrkräften in Slowenien mithilfe einer schriftlichen Befragung zu erfahren, was für eine Rolle Grammatik in ihrem DaF-Unterricht spielt, wie sie grammatische Strukturen vermitteln und was dabei die Orientierung darstellt. Es werden zunächst wichtige Aspekte aus den Fachdiskussionen zum Thema geschildert. Des Weiteren wird die Rolle der Grammatik im aktuellen slowenischen Nationalcurriculum für Deutsch als Fremdsprache in der Grundschule und am Gymnasium sowie im Abiturkatalog dargestellt. Schließlich folgt der empirische Teil mit Präsentation der Forschungsergebnisse. 


\section{DER STELLENWERT DER GRAMMATIK IM FREMDSPRACHENUNTERRICHT}

Es bestehen unterschiedliche Auflistungen von didaktisch-methodischen Prinzipien bzw. Unterrichtsprinzipien. Als die Unterrichtsprinzipien des heutigen Sprachunterrichts hebt Klippel (2016: 317-319) die Kompetenz- und Handlungsorientierung, Selbsttätigkeit und Authentizität hervor. Grotjahn \& Kleppin (2013: 26) führen neben der Handlungsund Kompetenzorientierung sowie der Lernerorientierung und -aktivierung die Interaktionsorientierung, Förderung von autonomem Lernen, interkulturelle Orientierung, Mehrsprachigkeits- und Aufgabenorientierung an. Von der Fort- und Weiterbildungsreihe Deutsch lehren lernen des Goethe-Instituts (2013) werden für die Deutschvermittlung insgesamt zehn didaktisch-methodische Prinzipien empfohlen: Kompetenzorientierung, Lernerorientierung, Handlungsorientierung, Lerneraktivierung, Interaktionsorientierung, Förderung der Lernerautonomie, interkulturelle Orientierung, Aufgabenorientierung, Mehrsprachigkeitsorientierung sowie die Integration digitaler Medien.

Versteht man die kommunikative Kompetenz als das oberste Ziel des Fremdsprachenunterrichts, so wäre von den didaktisch-methodischen Prinzipien die Handlungsorientierung hervorzuheben. Um das Ziel, dass Lernende mit der Sprache handeln können, zu erreichen, schlagen Funk et al. (2014) anstatt des traditionellen Modells von vier Fertigkeiten sowie den separaten Komponenten Wortschatz und Grammatik in Anlehnung an Nation \& Newton (nach Funk et al. 2014: 22-23) das sog. Modell von vier Lernfeldern vor: Arbeit mit bedeutungsvollen Inhalten (Lernen durch Hören, Lesen, Hörsehen), Arbeit mit sprachlichen Formen (sprachformbezogenes Lernen), Produktion von bedeutungsvollem Output (Lernen durch Sprechen und Schreiben) und Training von Flüssigkeit (flüssig werden im Sprechen, Schreiben, Hören, Lesen, Hörsehen).

Das Lernfeldermodell geht dabei davon aus, dass die Lernaktivitäten im Rahmen eines gesamten Kurses bzw. Schuljahres alle vier Lernfelder im gleichen Umfang abdecken sollen, so dass eine ausgeglichene Kompetenzentwicklung gewährleistet wird (Funk et al. 2014: 23). Die gleichmäßige Verteilung der Aktivitäten wird dabei mit dem Time-on-Task-Prinzip begründet, nach dem die zur Verfügung stehende Zeit darauf verwendet wird, was die Lernenden später können sollen, denn „Lesen lernt man nur durch Lesen, Sprechen durch Sprechen, und Flüssigkeit kann in einer Sprache nur erreicht werden, wenn man sie auch trainiert" (Funk et al. 2014: 23). Sich im DaF-Unterricht auch mit Grammatik zu beschäftigen, ist allerdings nicht verboten, sondern empfehlenswert. Nach Funk et al. (2014: 24) bedeutet die Arbeit mit sprachlichen Formen als einem der vier Lernfelder nämlich ,eine zeitweise Konzentration auf sprachliche Strukturen auf der Wort-, Satz- und Textebene im Rahmen eines inhalts- und handlungsorientierten Fremdsprachenunterrichts".

Im Allgemeinen werden in der heutigen Fachdiskussion kognitive Lerntheorien, wie es Drumm et al. (2013: 20) konstatieren, am stärksten gewichtet. Man muss Funk et al. 
(2014: 23) jedoch völlig zustimmen, dass, ,sich mündlich-produktive Kompetenz, also das flüssige Sprechen einer fremden Sprache, nicht als Nebenprodukt der bewusst gemachten Regeln durch das Üben grammatischer Formen einstellt“". Gnutzmann (2016: 146) hebt mehrfach hervor, dass die Rolle des expliziten, bewussten Wissens im Vergleich zu der des impliziten, unbewussten Wissens weniger eindeutig ist. Zu den Untersuchungen über die Wirkung sprachlicher Bewusstmachung von ausgewählten grammatischen Erscheinungen im Fremdsprachenunterricht merkt er kritisch an, ,dass sie sich im Allgemeinen nur über kurze Zeiträume erstrecken“; er gibt zu, dass ein positiver Zusammenhang von explizitem Wissen und Kompetenzentwicklung festgestellt werden kann, es bleibt aber seinen Worten nach ,aufgrund fehlender Langzeitstudien offen, ob die gelernte sprachliche Regel ebenfalls langfristig verfügbar ist" (Gnutzmann 2016: 146).

Die Diskrepanz zwischen Wissen einerseits und Können andererseits lässt sich in der Unterrichtspraxis tatsächlich häufig feststellen. Dennoch schließen sich in dem oben dargestellten Lernfeldermodell bewusstmachende und automatisierende Unterrichtsverfahren nicht aus. Vielmehr wird die Arbeit mit sprachlichen Formen durch das Training von Flüssigkeit ergänzt.

\section{3}

\section{DIE GRAMMATIK IM SLOWENISCHEN NATIONALCURRICULUM UND ABITURKATALOG}

Das aktuelle slowenische Nationalcurriculum für Deutsch als erste Fremdsprache in der Grundschule (4.-9. Klasse) gibt als Ziel des Deutschunterrichts die kommunikative Kompetenz an, die sich aus der sprachlichen, pragmatischen und soziokulturellen Kompetenz zusammensetzt (Kač et al. 2016: 11). Der Deutschunterricht soll den Schülern und Schülerinnen die sprachlichen Mittel (Wortschatz, Redewendungen, Strukturen, Aussprache, Intonation, Rechtschreibung, Grammatik) vermitteln sowie Lerntechniken und -strategien und soll sie darüber hinaus zur Rezeption und Produktion in der Zielsprache befähigen (Kač et al. 2016: 11). Es wird betont, dass in der 4. und 5. Klasse eine systematische Entwicklung der grammatischen Kompetenz von untergeordneter Bedeutung sein soll (Kač et al. 2016: 37).

Laut dem slowenischen Nationalcurriculum für Deutsch als Wahlpflichtfach in der Grundschule (7.-9. Klasse) beginnen die Schüler und Schülerinnen in der Grundschule die interkulturelle und intersprachliche kommunikative Kompetenz zu entwickeln, indem sie alle vier sprachlichen Fertigkeiten entwickeln, d. h. das Hören, Lesen, Sprechen und Schreiben (Kondrič Horvat et al. 2001: 7). Dafür entwickeln sie die sprachliche, soziolinguistische, diskursive und soziokulturelle Fähigkeit sowie die kommunikativen Strategien und das selbstständige Lernen (Kondrič Horvat et al. 2001: 9).

Nach dem slowenischen Nationalcurriculum für die zweite Fremdsprache als Wahlfach in der Grundschule (4.-9. Klasse) ist das allgemeine Ziel dieses Unterrichtes die 
Entwicklung der kommunikativen Kompetenz, die sich aus der sprachlichen, pragmatischen und soziokulturellen Kompetenz zusammensetzt (Pevec Semec et al. 2013: 5). Von der 4. bis zur 6. Klasse sollen die Rezeption und Interaktion im Vordergrund stehen; das Schreiben soll sich auf einzelne einfache Wörter und Sätze beschränken (Pevec Semec et al. 2013: 8). Ab der 7. Klasse sollen dann alle Fertigkeiten entwickelt werden, die Lehrkräfte sollen die Grammatik aber nicht explizit erklären, sondern sie in unterschiedlichen Situationen befestigen (Pevec Semec et al. 2013: 10).

Laut dem aktuellen slowenischen Nationalcurriculum für Deutsch als Fremdsprache am Gymnasium (Holc et al. 2008: 10) erreichen die Schüler und Schülerinnen die kommunikative Kompetenz im Deutschen als Fremdsprache, indem sie die rezeptiven und produktiven Kompetenzen sowie die Interaktion, Sprachmittlung und die interkulturelle Kompetenz ausbauen. Dabei entwickeln sie spezifische sprachliche Fertigkeiten, darunter die sprachlichen Fertigkeiten im engeren Sinne, d. h., sie erlernen den Wortschatz, die Orthografie und Orthoepie, die Morphologie und Syntax (Holc et al. 2008: 10). Des Weiteren werden im Nationalcurriculum die zu behandelnden Strukturen aufgelistet und beschrieben.

Noch genauer sind die erwarteten Strukturen aus den Bereichen Morphologie und Syntax für das Grundniveau sowie das höhere Niveau der slowenischen Abiturprüfung im Fach Deutsch als Fremdsprache im jeweiligen Abiturkatalog beschrieben. Bei den Prüfungszielen wird bei beiden Niveaus angegeben, der Kandidat bzw. die Kandidatin solle zeigen, dass er/ sie die erwarteten Strukturen gebrauchen kann (Gliha Olenik et al. 2018: 6). Dies wird im Teil Sprachgebrauch direkt sowie in den Teilen Schreiben und Sprechen indirekt überprüft. Während in den Teilen Schreiben und Sprechen die sprachliche Korrektheit nur eines der Kriterien darstellt, werden die sprachlichen Elemente im Teil Sprachgebrauch im Kontext geprüft; die Kandidaten und Kandidatinnen müssen diese entsprechend auswählen, ergänzen bzw. umformen. Dies stellt $15 \%$ der Gesamtnote dar, die Teile Schreiben und Sprechen weitere $30 \%$ bzw. $20 \%$ (Lese- und Hörverstehen hingegen $20 \%$ bzw. $15 \%$ ). Rechnet man zur Bewertung des Teils Sprachgebrauch die Bewertung der sprachlichen Korrektheit im Rahmen der Schreib- und Sprechkompetenz hinzu, so wird klar, dass die grammatische Kompetenz bei der slowenischen Abiturprüfung keine unwichtige Rolle spielt.

\section{$4 \quad$ EMPIRISCHER TEIL}

\subsection{Forschungsproblem}

In der Geschichte der Fremdsprachendidaktik wurde der Grammatik als Teilkompetenz ein äußerst unterschiedlicher Stellenwert zugeschrieben. Doch auch im modernen effizienz- und handlungsorientierten Deutsch-als-Fremdsprache-Unterricht bleibt die Rolle der Grammatik umstritten, weshalb auch die Verfahren in der Unterrichtspraxis unterschiedlich 
bleiben. Das Ganze zeigt sich u. a. in den internationalen und nationalen Prüfungsformaten: In einigen - etwa Goethe-Zertifikat $\mathrm{C} 1$ und $\mathrm{C} 2$ - wird Grammatik als Teilkompetenz direkt geprüft und bewertet, in anderen (Goethe-Zertifikate von A1 bis B2; Deutsches Sprachdiplom I und II) hingegen nur im Rahmen anderer Kompetenzen. Da die slowenische Abiturprüfung im Fach Deutsch sowohl auf dem Niveau B1 als auch B2 Grammatik nicht nur indirekt, sondern auch direkt prüft, stellen sich die Fragen, was für eine Rolle Grammatik im institutionalisierten Deutschunterricht in Slowenien spielt und wie sie vermittelt wird.

\subsection{Methode und Instrument}

Wir bedienten uns der quantitativen Forschung und haben eine schriftliche Befragung der Lehrkräfte vorgesehen, für die ein Fragebogen mit sechs geschlossenen Fragen mit jeweils einer möglichen Antwort sowie drei zusätzlichen Fragen zu demografischen Daten (Geschlecht, Dauer und Art der Berufserfahrung) entwickelt wurde. Zum Ausfüllen des Fragebogens waren fünf Minuten vorgesehen.

\subsection{Datenerhebung und Datenaufbereitung}

Der Fragebogen wurde zunächst an externe Bewerter und Bewerterinnen der slowenischen Abiturprüfung im Fach Deutsch als Fremdsprache an ihrem jährlichen Treffen verteilt. Dies ist eine spezifische Gruppe, die sich in erster Linie aus aktuellen, aber auch vereinzelten ehemaligen gymnasialen DaF-Lehrkräften zusammensetzt. Um einen Vergleich zu ermöglichen, wurde der Fragebogen danach - aufgrund der epidemischen Situation diesmal digital als Online-Umfrage - an weitere slowenische DaF-Lehrkräfte, die an Webinaren der Autorin teilnahmen, aber keine externen Bewerter und Bewerterinnen der slowenischen Abiturprüfung im Fach Deutsch als Fremdsprache sind, vermittelt. Die Daten aus den Fragebögen wurden in Excel übertragen und mithilfe der deskriptivstatistischen Verfahren analysiert, wobei die Mittelwerte (MT), Standardabweichungen (STD) und Prozentzahlen (f \%) berechnet wurden.

\subsection{Teilnehmende}

An der Befragung nahmen in der ersten Gruppe 40 externe Bewerter und Bewerterinnen der slowenischen Abiturprüfung als Deutschlehrkräfte aus Slowenien teil, davon 39 Frauen und ein Mann. Die Stichprobe $(\mathrm{N}=40)$ ist im Allgemeinen klein und die Gruppe spezifisch, so dass die Ergebnisse nicht auf die Gesamtpopulation der Deutschlehrenden an slowenischen Gymnasien verallgemeinerbar sind, doch wenn man bedenkt, dass es in Slowenien insgesamt nur etwa 60 Gymnasien gibt, ermöglichen sie doch einen Einblick 
in die vorherrschende Praxis und scheinen auf reale Lehr-Lern-Kontexte übertragbar zu sein. In der zweiten Gruppe nahmen an der Befragung noch zusätzliche 22 Deutschlehrkräfte aus Slowenien teil, die an verschiedenen Institutionen Deutsch als Fremdsprache unterrichten, aber keine externen Bewerter und Bewerterinnen der slowenischen Abiturprüfung sind; darunter waren lediglich Frauen.

Tabelle 1.1 zeigt, dass die meisten, also 62,5\% der teilnehmenden Lehrkräfte aus der ersten Gruppe, zwischen 16 und 25 Jahre Berufserfahrung haben. $30 \%$ der Teilnehmenden verfügen über mehr als 25 Jahre Berufserfahrung, zwei Teilnehmende über 11-15 Jahre und nur eine über fünf Jahre oder weniger, allerdings wenigstens über ein Jahr. Dies bedeutet, dass wir es generell mit erfahrenen DaF-Lehrkräften zu tun haben, welche die anfänglichen Schwierigkeiten wohl schon gemeistert haben.

Tabelle 1.1: Berufserfahrung der Teilnehmenden aus der ersten Gruppe

\begin{tabular}{|l|l|r|r|}
\hline Fr. $\mathbf{1}$ & $\begin{array}{l}\text { Über wie viele Jahre Berufserfahrung im Bereich Deutsch-als- } \\
\text { Fremdsprache-Unterricht verfügen Sie? }\end{array}$ & $\mathbf{f} \%$ \\
\hline e) & 16-25 Jahre & 25 & 62,5 \\
\hline f) & mehr als 25 Jahre & 12 & 30,0 \\
\hline d) & 11-15 Jahre & 2 & 5,0 \\
\hline b) & 1-5 Jahre & 1 & 2,5 \\
\hline a) & weniger als 1 Jahr & 0 & 0,0 \\
\hline c) & 6-10 Jahre & 0 & 0,0 \\
\hline Gesamt & 40 & 100,0 \\
\hline
\end{tabular}

Tabelle 1.2 zeigt, dass die meisten, also 45,5\% der teilnehmenden Lehrkräfte aus der zweiten Gruppe, zwischen 11 und 15 Jahre Berufserfahrung haben. 22,7 \% der Teilnehmenden haben zwischen 6 und 10 Jahre und 18,2\% zwischen 16 und 25 Jahre

Tabelle 1.2: Berufserfahrung der Teilnehmenden aus der zweiten Gruppe

\begin{tabular}{|l|l|r|r|}
\hline Fr. $\mathbf{1}$ & $\begin{array}{l}\text { Über wie viele Jahre Berufserfahrung im Bereich Deutsch-als- } \\
\text { Fremdsprache-Unterricht verfügen Sie? }\end{array}$ & $\mathbf{f} \%$ \\
\hline d) & 11-15 Jahre & 10 & 45,5 \\
\hline c) & 6-10 Jahre & 5 & 22,7 \\
\hline e) & 16-25 Jahre & 4 & 18,2 \\
\hline f) & mehr als 25 Jahre & 2 & 9,1 \\
\hline b) & 1-5 Jahre & 1 & 4,5 \\
\hline a) & weniger als 1 Jahr & 0 & 0,0 \\
\hline Gesamt & 22 & 100,0 \\
\hline
\end{tabular}


Berufserfahrung, zwei Teilnehmende verfügen über mehr als 25 Jahre Berufserfahrung und nur eine über fünf Jahre oder weniger, allerdings wenigstens über ein Jahr. Im Vergleich zur ersten Gruppe haben wir es hier mit etwas weniger erfahrenen Lehrerinnen zu tun, jedoch auch nicht Anfängerinnen.

Tabelle 2.1 ist zu entnehmen, dass 72,5\% der Teilnehmenden aus der ersten Gruppe an einem Gymnasium unterrichten und 27,5 \% an anderen Schularten: Sechs Teilnehmende unterrichten an einer Fachmittelschule und zwei an einer Grundschule, jeweils eine unterrichtet an einer Fakultät oder einer Sprachschule, während eine Teilnehmende DaF zurzeit nicht unterrichtet. Sowohl an Gymnasien und Fachmittelschulen als auch an Grundschulen kann Deutsch als Fremdsprache bei nationalen Prüfungen geprüft werden, allerdings wird Grammatik im Unterschied zu der allgemeinen Abiturprüfung und der nationalen Grundschulprüfung im Berufsabitur nicht direkt geprüft.

\section{Tabelle 2.1: Arbeitsstelle der Teilnehmenden aus der ersten Gruppe}

\begin{tabular}{|l|l|r|r|}
\hline Fr. 2 & Wo unterrichten Sie Deutsch als Fremdsprache hauptsächlich? & F & f \% \\
\hline d) & an einem Gymnasium & 29 & 72,5 \\
\hline c) & an einer Fachmittelschule & 6 & 15,0 \\
\hline a) & an einer Grundschule & 2 & 5,0 \\
\hline e) & an einer Fakultät & 1 & 2,5 \\
\hline f) & an einer Sprachschule & 1 & 2,5 \\
\hline g) & Ich unterrichte zurzeit DaF nicht (mehr). & 1 & 2,5 \\
\hline b) & an einer Berufsschule & 0 & 0,0 \\
\hline Gesamt & 40 & 100,0 \\
\hline
\end{tabular}

Tabelle 2.2 ist zu entnehmen, dass 68,2 \% der Teilnehmenden aus der zweiten Gruppe an einer Grundschule unterrichten und 31,8 \% an anderen Schularten: Drei Teilnehmende

Tabelle 2.2: Arbeitsstelle der Teilnehmenden aus der zweiten Gruppe

\begin{tabular}{|l|l|r|r|}
\hline Fr. 2 & Wo unterrichten Sie Deutsch als Fremdsprache hauptsächlich? & F & $\mathbf{f} \%$ \\
\hline a) & an einer Grundschule & 15 & 68,2 \\
\hline d) & an einem Gymnasium & 3 & 13,6 \\
\hline f) & an einer Sprachschule & 3 & 13,6 \\
\hline e) & an einer Fakultät & 1 & 4,6 \\
\hline c) & an einer Fachmittelschule & 0 & 0,0 \\
\hline g) & Ich unterrichte zurzeit DaF nicht (mehr). & 0 & 0,0 \\
\hline b) & an einer Berufsschule & 0 & 0,0 \\
\hline Gesamt & 22 & 100,0 \\
\hline
\end{tabular}


unterrichten an einem Gymnasium, weitere drei an einer Sprachschule, während eine an einer Fakultät unterrichtet. Während in der ersten Gruppe vor allem DaF-Lehrkräfte an Gymnasien vertreten sind, setzt sich die Mehrheit der Teilnehmenden in der zweiten Gruppe demnach aus Grundschullehrerinnen zusammen.

\subsection{Forschungsergebnisse und Diskussion}

Mit der dritten Frage sollte herausgefunden werden, wie die Teilnehmenden die Bedeutung der Grammatikkenntnisse zur Erreichung der kommunikativen Kompetenz im Deutschen als Fremdsprache einschätzen. Tabelle 3.1 kann entnommen werden, dass $55 \%$ der Teilnehmenden aus der ersten Gruppe auf der Skala von 1 bis 5 (1-gar nicht wichtig, 5 - sehr wichtig) die Bedeutung mit 4 bewertet haben, während sich jeweils 22,5\% für den Wert 3 bzw. 5 entschieden haben $(\mathrm{MT}=4,0)$. Die Werte 1 und 2 hingegen wurden nicht vergeben.

Tabelle 3.1: Wichtigkeit der Grammatikkenntnisse für die kommunikative Kompetenz, Gruppe 1

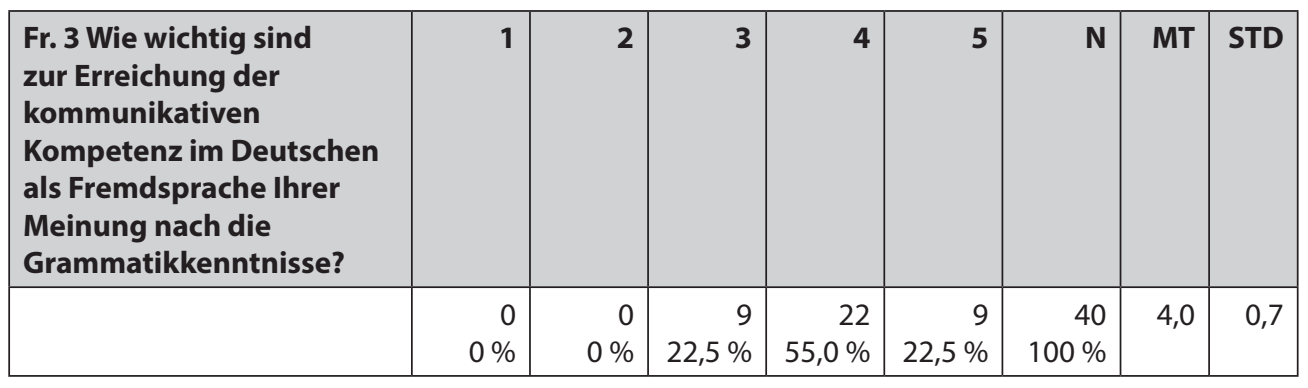

Tabelle 3.2 kann entnommen werden, dass 54,6\% der Teilnehmenden aus der zweiten Gruppe die Bedeutung ebenso mit 4 bewertet haben; 40,9 \% haben sich allerdings für den Wert 3 entschieden und nur eine Teilnehmende für $5(\mathrm{MT}=3,6)$. Die Werte 1 und 2 wurden auch in dieser Gruppe nicht vergeben. Insgesamt wurden Grammatikkenntnisse von den teilnehmenden Lehrkräften aus beiden Gruppen also als wichtig eingeschätzt, in der ersten Gruppe jedoch als etwas wichtiger als in der zweiten. Der Wert 3 wurde dabei in der zweiten Gruppe von sieben Grundschullehrerinnen vergeben sowie von jeweils einer Lehrerin an der Fakultät bzw. in der Sprachschule. Die vertretenen Grundschullehrerinnen schätzen also Grammatik als etwas weniger wichtig zur Erreichung der kommunikativen Kompetenz ein als die teilnehmenden DaF-Lehrerinnen an Gymnasien.

Mit der vierten Frage wollten wir erfahren, was für eine Rolle Grammatik im Vergleich zu anderen (Teil)Kompetenzen im DaF-Unterricht der Teilnehmenden spielt. Tabelle 4.1 zeigt, dass sich die Teilnehmenden aus der ersten Gruppe zu $75 \%$ für die 
Tabelle 3.2: Wichtigkeit der Grammatikkenntnisse für die kommunikative Kompetenz, Gruppe 2

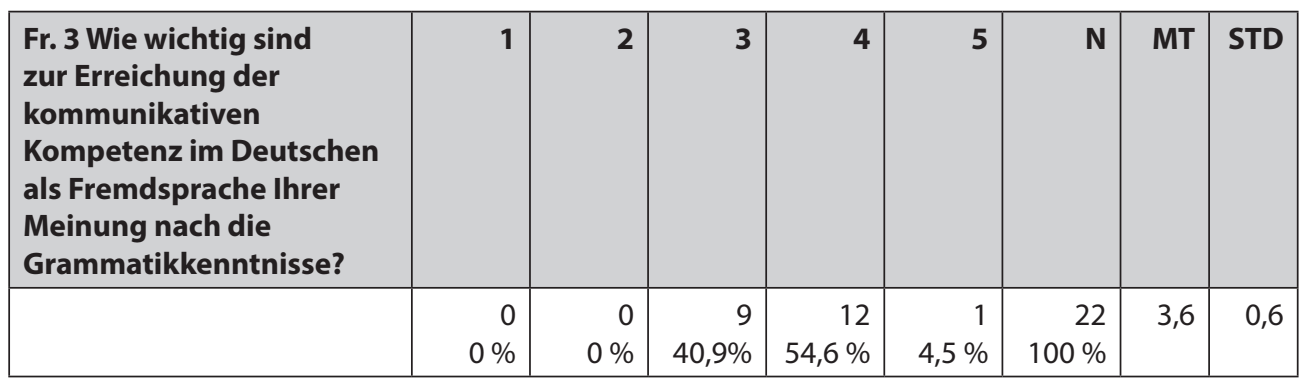

Antwort eine gleichwertige wie die anderen Teilfertigkeiten entschieden haben. An zweiter Stelle befindet sich mit $15 \%$ die Antwort eine kleinere als die anderen Teilfertigkeiten und an dritter Stelle mit $10 \%$ die Antwort eine zentrale. Die Antworten eine sehr geringe und gar keine wurden von den Teilnehmenden nicht ausgewählt. Die große Mehrheit der Teilnehmenden versteht die grammatische also als eine den anderen Teilfertigkeiten gleichrangige Kompetenz.

Tabelle 4.1: Rolle der Grammatik im DaF-Unterricht, Gruppe 1

\begin{tabular}{|l|l|r|r|}
\hline Fr. $\mathbf{4}$ & Was für eine Rolle spielt Grammatik in Ihrem DaF-Unterricht? & F & f\% \\
\hline b) & eine gleichwertige wie die anderen Teilfertigkeiten & 30 & 75,0 \\
\hline c) & eine kleinere als die anderen Teilfertigkeiten & 6 & 15,0 \\
\hline a) & eine zentrale & 4 & 10,0 \\
\hline d) & eine sehr geringe & 0 & 0,0 \\
\hline e) & gar keine & 0 & 0,0 \\
\hline \multicolumn{2}{|l|}{ Gesamt } & 40 & 100,0 \\
\hline
\end{tabular}

Tabelle 4.2 zeigt, dass sich die Teilnehmenden aus der zweiten Gruppe zu 54,5 \% für die Antwort eine gleichwertige wie die anderen Teilfertigkeiten entschieden haben. An zweiter Stelle befindet sich mit 36,4 \% auch hier die Antwort eine kleinere als die anderen Teilfertigkeiten und an dritter Stelle mit 9,1\% die Antwort eine zentrale. Die Antworten eine sehr geringe und gar keine wurden von den Teilnehmenden aus der zweiten Gruppe ebenso nicht ausgewählt. Auch die Mehrheit der Teilnehmenden aus der zweiten Gruppe versteht die grammatische als eine den anderen Teilfertigkeiten gleichrangige Kompetenz; die Zahl derjenigen, die der Grammatik eine kleinere Rolle als den anderen Teilfertigkeiten verteilen, ist hier im Vergleich zu der ersten Gruppe allerdings größer. Diese Antwort wurde auch hier vor allem von der Grundschullehrerinnen gewählt (sechs Teilnehmende), dafür haben sich aber auch zwei Lehrerinnen von den Sprachschulen entschieden. 
Tabelle 4.2: Rolle der Grammatik im DaF-Unterricht, Gruppe 2

\begin{tabular}{|l|l|r|r|}
\hline Fr. $\mathbf{4}$ & Was für eine Rolle spielt Grammatik in Ihrem DaF-Unterricht? & F & $\mathbf{f} \%$ \\
\hline b) & eine gleichwertige wie die anderen Teilfertigkeiten & 12 & 54,5 \\
\hline c) & eine kleinere als die anderen Teilfertigkeiten & 8 & 36,4 \\
\hline a) & eine zentrale & 2 & 9,1 \\
\hline d) & eine sehr geringe & 0 & 0,0 \\
\hline e) & gar keine & 0 & 0,0 \\
\hline Gesamt & 22 & 100,0 \\
\hline
\end{tabular}

Mit der fünften Frage sollte festgestellt werden, welche Verfahren die Teilnehmenden zur Vermittlung von Grammatik im DaF-Unterricht anwenden. Tabelle 5.1 kann entnommen werden, dass dabei die meisten Teilnehmenden aus der ersten Gruppe (60\%) eine Kombination von automatisierenden und kognitivierenden Verfahren und 37,5\% nur die kognitivierenden Verfahren (Analyse, Erklärung, Bewusstmachung) ausgewählt haben. Für ausschließlich automatisierende Verfahren (Erwerben von Chunks ohne Bewusstmachung) hat sich lediglich eine Person entschieden, die an einem Gymnasium unterrichtet; der kleine Anteil dieser Antworten überrascht jedoch nicht ganz, denn die Gymnasiasten und Gymnasiastinnen, welche die meisten an dieser Befragung Teilnehmenden in der Praxis unterrichten, verfügen auch in anderen (Fremd)Sprachen über Kenntnisse, was im Sinne der Mehrsprachigkeitsorientierung zu Sprachvergleichen und Analysen führen kann.

Tabelle 5.1: Art der Vermittlung von Grammatik im DaF-Unterricht, Gruppe 1

\begin{tabular}{|l|l|r|r|}
\hline Fr. $\mathbf{5}$ & $\begin{array}{l}\text { Wie vermitteln Sie in Ihrem DaF-Unterricht die grammatischen } \\
\text { Strukturen? }\end{array}$ & $\mathbf{f} \%$ \\
\hline c) & sowohl mit automatisierenden als auch kognitivierenden Verfahren & 24 & 60,0 \\
\hline b) & mit kognitivierenden Verfahren (Analyse, Erklärung, Bewusstmachung) & 15 & 37,5 \\
\hline a) & $\begin{array}{l}\text { mit automatisierenden Verfahren (Erwerben von Chunks ohne } \\
\text { Bewusstmachung) }\end{array}$ & 1 & 2,5 \\
\hline \multicolumn{2}{|l|}{ Gesamt } & 40 & 100,0 \\
\hline
\end{tabular}

Tabelle 5.2 kann entnommen werden, dass die meisten Teilnehmenden aus der zweiten Gruppe (86,4 \%) ebenso eine Kombination von automatisierenden und kognitivierenden Verfahren und 9,1\% nur die kognitivierenden Verfahren ausgewählt haben. Für ausschließlich automatisierende Verfahren hat sich auch in dieser Gruppe lediglich eine Person entschieden, die an einer Grundschule unterrichtet. Lediglich kognitivierende Verfahren werden von dieser Gruppe DaF-Lehrerinnen also wesentlich weniger angewendet, als dies in der ersten Gruppe der Fall war. 
Tabelle 5.2: Art der Vermittlung von Grammatik im DaF-Unterricht, Gruppe 2

\begin{tabular}{|l|l|r|r|}
\hline Fr. $\mathbf{5}$ & $\begin{array}{l}\text { Wie vermitteln Sie in Ihrem DaF-Unterricht die grammatischen } \\
\text { Strukturen? }\end{array}$ & $\mathbf{f} \%$ \\
\hline c) & sowohl mit automatisierenden als auch kognitivierenden Verfahren & 19 & 86,4 \\
\hline b) & mit kognitivierenden Verfahren (Analyse, Erklärung, Bewusstmachung) & 2 & 9,1 \\
\hline a) & $\begin{array}{l}\text { mit automatisierenden Verfahren (Erwerben von Chunks ohne } \\
\text { Bewusstmachung) }\end{array}$ & 1 & 4,5 \\
\hline \multicolumn{2}{|l|}{ Gesamt } & 22 & 100,0 \\
\hline
\end{tabular}

Mit der sechsten Frage wollten wir herausfinden, in welcher Sprache die Teilnehmenden in ihrem DaF-Unterricht grammatische Phänomene erklären. Wie Tabelle 6.1 zeigt, gaben $60 \%$ der Teilnehmenden aus der ersten Gruppe dabei eine Kombination der Erst- und Zielsprache an und 35 \% nur die Erstsprache. Zwei Personen, die beide am Gymnasium unterrichten, haben lediglich Deutsch als Zielsprache ausgewählt. Diese Resultate entsprechen den Antworten auf die fünfte Frage, d. i. welche Verfahren sie zur Vermittlung von Grammatik in ihrem DaF-Unterricht verwenden, bei denen sich die Teilnehmenden zu 60 \% für eine Kombination von automatisierenden und kognitivierenden Verfahren und zu 37,5 \% für ausschließlich kognitivierende Verfahren entschieden haben; während Kognitivierung insbesondere auf niedrigeren Niveaustufen wohl in der Erstsprache durchgeführt werden muss, kann Automatisierung nicht anders als in der Zielsprache erfolgen.

Tabelle 6.1: Sprache der Vermittlung von Grammatik im DaF-Unterricht, Gruppe 1

\begin{tabular}{|l|l|r|r|}
\hline Fr. 6 & $\begin{array}{l}\text { In welcher Sprache erklären Sie grammatische Phänomene der } \\
\text { deutschen Sprache in Ihrem Unterricht? }\end{array}$ & f \% \\
\hline c) & in einer Kombination der Erst- und Zielsprache & 24 & 60,0 \\
\hline a) & in der Erstsprache der Mehrheit & 14 & 35,0 \\
\hline b) & im Deutschen als der Zielsprache & 2 & 5,0 \\
\hline d) & in einer anderen allen gemeinsamen Fremdsprache & 0 & 0,0 \\
\hline e) & Ich erkläre keine grammatischen Phänomene. & 0 & 0,0 \\
\hline Gesamt & 40 & 100,0 \\
\hline
\end{tabular}

Wie Tabelle 6.2 zeigt, gaben 72,7\% der Teilnehmenden aus der zweiten Gruppe eine Kombination der Erst- und Zielsprache an und 27,3 \% nur die Erstsprache. Während diese Resultate mit deren aus der ersten Gruppe vergleichbar sind, wurde lediglich Deutsch als Zielsprache hier anders als in der ersten Gruppe aber gar nicht ausgewählt. 
Tabelle 6.2: Sprache der Vermittlung von Grammatik im DaF-Unterricht, Gruppe 2

\begin{tabular}{|l|l|r|r|}
\hline Fr. $\mathbf{6}$ & $\begin{array}{l}\text { In welcher Sprache erklären Sie grammatische Phänomene der } \\
\text { deutschen Sprache in Ihrem Unterricht? }\end{array}$ & $\mathbf{f} \%$ \\
\hline c) & in einer Kombination der Erst- und Zielsprache & 16 & 72,7 \\
\hline a) & in der Erstsprache der Mehrheit & 6 & 27,3 \\
\hline b) & im Deutschen als der Zielsprache & 0 & 0,0 \\
\hline d) & in einer anderen allen gemeinsamen Fremdsprache & 0 & 0,0 \\
\hline e) & Ich erkläre keine grammatischen Phänomene. & 0 & 0,0 \\
\hline \multicolumn{2}{|l|}{ Gesamt } & 22 & 100,0 \\
\hline
\end{tabular}

Mit der siebten Frage wollten wir erfahren, an welchen Richtlinien sich die Teilnehmenden bei der Einbeziehung von Grammatik in ihren DaF-Unterricht hauptsächlich orientieren. Tabelle 7.1 ist zu entnehmen, dass sich die Antwort an eigenen Erfahrungen und Erkenntnissen mit $40 \%$ in der ersten Gruppe an erster Stelle befindet, gefolgt von den Antworten an dem benutzten Lehrwerk mit $25 \%$, an dem aktuellen Nationalcurriculum mit $15 \%$ und an dem Maturakatalog ${ }^{1}$ mit 12,5\%. Für die Antwort an dem Gemeinsamen Europäischen Referenzrahmen für Sprachen haben sich drei Personen entschieden.

Tabelle 7.1: Orientierung bei der Vermittlung von Grammatik im DaF-Unterricht, Gruppe 1

\begin{tabular}{|l|l|r|r|}
\hline Fr. 7 & $\begin{array}{l}\text { Woran orientieren Sie sich bei der Einbeziehung der Grammatik in Ihren } \\
\text { DaF-Unterricht hauptsächlich? }\end{array}$ & $\mathbf{f} \%$ \\
\hline b) & an eigenen Erfahrungen und Erkenntnissen & 16 & 40,0 \\
\hline a) & an dem benutzten Lehrwerk & 10 & 25,0 \\
\hline e) & an dem aktuellen Nationalkurrikulum & 6 & 15,0 \\
\hline d) & an dem Maturakatalog & 5 & 12,5 \\
\hline c) & an dem Gemeinsamen Europäischen Referenzrahmen für Sprachen & 3 & 7,5 \\
\hline \multicolumn{2}{|l|}{ Gesamt } & 40 & 100,0 \\
\hline
\end{tabular}

Tabelle 7.2 ist zu entnehmen, dass sich in der zweiten Gruppe an erster Stelle mit 63,6 \% die Antwort an dem benutzten Lehrwerk befindet, gefolgt von der Antwort an eigenen Erfahrungen und Erkenntnissen mit 27,2 \%. Die Antwort an dem aktuellen Nationalcurriculum bzw. an dem Maturakatalog hat in dieser Gruppe jeweils eine Person gewählt. Für die Antwort an dem Gemeinsamen Europäischen Referenzrahmen für Sprachen hat sich keine entschieden.

\footnotetext{
1 Im Slowenischen heißt das Abitur matura und weil dieser Ausdruck auch in einem Teil des deutschsprachigen Raumes existiert, wurde in der Umfrage die unter slowenischen Deutschlehrkräften verbreitete Benennung Maturakatalog benutzt.
} 
Tabelle 7.2: Orientierung bei der Vermittlung von Grammatik im DaF-Unterricht, Gruppe 2

\begin{tabular}{|l|l|r|r|}
\hline Fr. 7 & $\begin{array}{l}\text { Woran orientieren Sie sich bei der Einbeziehung der Grammatik in Ihren } \\
\text { DaF-Unterricht hauptsächlich? }\end{array}$ & f \% \\
\hline a) & an dem benutzten Lehrwerk & 14 & 63,6 \\
\hline b) & an eigenen Erfahrungen und Erkenntnissen & 6 & 27,2 \\
\hline e) & an dem aktuellen Nationalkurrikulum & 1 & 4,6 \\
\hline d) & an dem Maturakatalog & 1 & 4,6 \\
\hline c) & an dem Gemeinsamen Europäischen Referenzrahmen für Sprachen & 0 & 0,0 \\
\hline \multicolumn{2}{|l|}{ Gesamt } & 22 & 100,0 \\
\hline
\end{tabular}

Der hohe Anteil der Antworten an eigenen Erfahrungen und Erkenntnissen in der ersten Gruppe könnte damit zusammenhängen, dass mehr als 90 \% der Teilnehmenden über 16 Jahre Berufserfahrung oder mehr verfügen. Die Häufigkeit der Antwort an dem benutzten Lehrwerk ist für keine Gruppe überraschend, wenn man bedenkt, dass an öffentlichen Schulen lediglich vom Schulamt zugelassene Lehrwerke gebraucht werden dürfen, die mit dem Nationalcurriculum übereinstimmen. Dass sich nur fünf Teilnehmende aus der ersten Gruppe für die Antwort an dem Maturakatalog entschieden haben, überrascht einerseits, andererseits sollte man aber bedenken, dass Deutsch kein Pflichtfach der Abiturprüfung ist, also kommt dieser in erster Linie bei der Abiturvorbereitung in Frage, die i. d. R. in getrennten Unterrichtsgruppen stattfindet.

Mit der letzten, achten Frage sollte dann der Einfluss der Überprüfung der grammatischen Kompetenz bei der Nationalprüfung Deutsch bzw. der Abiturprüfung Deutsch auf den DaF-Unterricht der Teilnehmenden festgestellt werden. Tabelle 8.1 zeigt, dass sich 57,5\% der Teilnehmenden aus der ersten Gruppe für die Antwort einen mittelgroßen Einfluss und $35 \%$ für die Antwort einen großen Einfluss entschieden haben. Die Antwort einen geringen Einfluss wurde von zwei Personen ausgewählt, von denen eine am Gymnasium und eine an der Fakultät unterrichtet, die Antwort keinen Einfluss wählte eine Person, die an einer Fachmittelschule unterrichtet.

Tabelle 8.1: Einfluss der Abiturprüfung Deutsch auf den DaF-Unterricht, Gruppe 1

\begin{tabular}{|l|l|r|r|}
\hline Fr. 8 & $\begin{array}{l}\text { Was für einen Einfluss hat die Überprüfung der grammatischen } \\
\text { Kompetenz bei der Nationalprüfung Deutsch bzw. der Abiturprüfung } \\
\text { Deutsch auf Ihren Unterricht? }\end{array}$ & F $\%$ \\
\hline b) & einen mittelgroßen Einfluss & 23 & 57,5 \\
\hline a) & einen großen Einfluss & 14 & 35,0 \\
\hline c) & einen geringen Einfluss & 2 & 5,0 \\
\hline d) & keinen Einfluss & 1 & 2,5 \\
\hline Gesamt & 40 & 100,0 \\
\hline
\end{tabular}


Tabelle 8.2 zeigt, dass sich 45,4 \% der Teilnehmenden aus der zweiten Gruppe ebenso für die Antwort einen mittelgroßen Einfluss entschieden haben, 27,3 \% aber für die Antwort keinen Einfluss. Die Antwort einen geringen Einfluss wurde von vier Personen ausgewählt, von denen zwei an Grundschulen und weitere zwei an Sprachschulen unterrichten, die Antwort einen großen Einfluss wählten zwei Personen, die an einer Grundschule unterrichten. Der im Vergleich zur ersten Gruppe hohe Anteil der Antworten keinen Einfluss kann damit zusammenhängen, dass Deutsch als (zweite) Fremdsprache als drittes Fach neben Erstsprache und Mathematik bei der Nationalprüfung Deutsch in der 9. Klasse Grundschule gar nicht jedes Jahr überprüft wird. Darüber hinaus ist es auch möglich, dass die teilnehmenden Lehrkräfte nur Deutsch als Wahlfach und nicht als Pflichtfach oder Wahlpflichtfach unterrichten.

Tabelle 8.2: Einfluss der Abiturprüfung Deutsch auf den DaF-Unterricht, Gruppe 2

\begin{tabular}{|l|l|r|r|}
\hline Fr. 8 & $\begin{array}{l}\text { Was für einen Einfluss hat die Überprüfung der grammatischen } \\
\text { Kompetenz bei der Nationalprüfung Deutsch bzw. der Abiturprüfung } \\
\text { Deutsch auf Ihren Unterricht? }\end{array}$ & f \% \\
\hline b) & einen mittelgroßen Einfluss & 10 & 45,4 \\
\hline d) & keinen Einfluss & 6 & 27,3 \\
\hline c) & einen geringen Einfluss & 4 & 18,2 \\
\hline a) & einen großen Einfluss & 2 & 9,1 \\
\hline Gesamt & 22 & 100,0 \\
\hline
\end{tabular}

In Verbindung mit der siebten Frage, bei der nur 12,5\% der Teilnehmenden aus der ersten Gruppe angegeben haben, dass sie sich bei der Einbeziehung der Grammatik in ihren DaF-Unterricht hauptsächlich am Abiturkatalog orientieren, erscheinen die Resultate bei dieser Frage auch in der ersten Gruppe auf den ersten Blick unlogisch. Allerdings sollte man nicht übersehen, dass die Schüler und Schülerinnen an den meisten Gymnasien erst am Ende der dritten Klasse die Wahlfächer für ihre Abiturprüfung auswählen, d. h. bis dahin werden alle zusammen unterrichtet. Und wenn am Ende des DaF-Unterrichtes eine Prüfung stattfindet, ist der Washback-Effekt dieser Prüfung auf den DaF-Unterricht bis zu einem bestimmten Grad natürlich auch zu erwarten - sowohl bei den Lehrkräften an Gymnasien als auch an den Grundschulen.

\section{5 \\ SCHLUSSBEMERKUNGEN}

In der vorliegenden Untersuchung wurde auf die Fragen eingegangen, was für eine Rolle Grammatik im DaF-Unterricht in Slowenien spielt und wie grammatische Strukturen vermittelt werden. Abschließend kann zusammengefasst werden, dass die Mehrheit der 
teilnehmenden Lehrenden aus beiden Gruppen, d. h. der teilnehmenden Abiturprüfenden sowie anderer teilnehmenden DaF-Lehrkräfte, Grammatikkenntnisse als wichtig für die Erreichung der kommunikativen Kompetenz im Deutschen als Zielsprache einschätzt und die Grammatik in ihrem Unterricht eine gleichwertige Rolle wie die anderen (Teil-) Kompetenzen einnimmt. Grammatische Strukturen vermitteln sie sowohl mithilfe automatisierender als auch kognitivierender Verfahren, die Erklärung erfolgt dabei meistens in einer Kombination der Erst- und Zielsprache. Bei der Einbeziehung der Grammatik in ihren DaF-Unterricht orientieren sich die teilnehmenden Lehrenden hauptsächlich an dem benutzten Lehrwerk und an eigenen Erfahrungen und Erkenntnissen.

In den Forschungsergebnissen kommen einige interessante Aspekte zum Ausdruck: In der ersten Gruppe haben die Teilnehmenden beispielsweise zu $40 \%$ angegeben, dass sie sich bei der Einbeziehung der Grammatik in ihren DaF-Unterricht hauptsächlich an eigenen Erfahrungen und Erkenntnissen orientieren. Dies kann durchaus mit der Tatsache zusammenhängen, dass 62,5\% der teilnehmenden Lehrkräfte aus dieser Gruppe über 16-25 Jahre und weitere 30 \% über mehr als 25 Jahre Berufserfahrung verfügen. Tatsächlich haben diese Antwort - mit einer Ausnahme, die zwischen 11-15 Jahre Berufserfahrung angegeben hat - lediglich erfahrene Lehrende ausgewählt, d. h. solche mit 16 Jahren Berufserfahrung oder mehr. Doch zugleich birgt diese Antwort eine Gefahr in sich, denn man weiß nicht, inwieweit diese Erkenntnisse mit den Entdeckungen auf dem Fachgebiet ausgebaut werden. Dass sich in dieser Gruppe mit 25\% an zweiter Stelle und in der zweiten Gruppe mit 63,6 \% an erster Stelle die Antwort an dem benutzten Lehrwerk befand, überrascht nicht, denn an öffentlichen Schulen dürfen lediglich vom Schulamt zugelassene Lehrwerke gebraucht werden, die mit dem Nationalcurriculum übereinstimmen.

Fast $40 \%$ der Teilnehmenden aus der ersten Gruppe (aber nur 9,1\% aus der zweiten Gruppe) vermitteln Grammatikkenntnisse lediglich mittels kognitivierender Verfahren. Dabei handelt es sich ausschließlich um Lehrkräfte mit 16 Jahren Berufserfahrung oder mehr, die bei der Orientierung aber unterschiedliche Antworten angegeben haben. Deutsch kommt im slowenischen Schulsystem zwar fast nur noch als zweite Fremdsprache vor und das bedeutet, dass die Schüler und Schülerinnen davor schon Erfahrungen mit der Erstsprache und einer ersten Fremdsprache, eventuell auch mit einer Zweitsprache gesammelt haben, was zu Sprachvergleichen und Analysen führen kann, die im Sinne der Mehrsprachigkeitsorientierung im modernen Fremdsprachenunterricht auch erwünscht sind. Allerdings klagen Lehrkräfte täglich darüber, dass ihre Lernenden die grammatischen Strukturen zwar kennen und verstehen, beim Schreiben und Sprechen aber dennoch viele Fehler machen. In einer Fremdsprache mit dem Kennen direkt zum Können zu gelangen, ist tatsächlich eher unwahrscheinlich. Laut dem dargestellten Lernfeldermodell benötigen die Lernenden zum Flüssigwerden das Automatisierungstraining.

Dass sich die Lehrkräfte der Bedeutung der Grammatikkenntnisse so sehr bewusst sind, obwohl das Lernen von Fremdsprachen heute im Allgemeinen äußerst effizienzorientiert ist, hat vermutlich auch mit dem Washback-Effekt der slowenischen 
Abiturprüfung im Fach Deutsch zu tun, in der im Teil Sprachgebrauch die grammatische Kompetenz im Sinne von Grammatik im Kontext geprüft und bewertet wird. Wäre dies - wie in vielen anderen internationalen Prüfungsformaten - nicht der Fall, so sähen auch die Resultate der vorliegenden Forschung wahrscheinlich etwas anders aus. Erfreulich ist allerdings, dass sich die Lehrenden trotz des lange geltenden Primats der Einsprachigkeit im Fremdsprachenunterricht inzwischen auch der Erstsprache der Mehrheit bedienen, um grammatische Phänomene zu erklären. Mit einem weiteren Schritt, dem Vergleichen, kann der Lernprozess im Tertiärsprachenunterricht wiederum effizienter und ökonomischer erfolgen.

Anders als das traditionelle Modell der vier Fertigkeiten integriert das einführend dargestellte Lernfeldermodell die Grammatik in das sprachliche Handeln und verleiht ihr einen neuen - verdienten - Stellenwert. Forschungsergebnisse zeigen, dass diesen auch viele teilnehmende Lehrkräfte (wieder)erkennen. Das Rätsel, wie die separaten Komponenten Grammatik und Wortschatz im Fremdsprachenunterricht mit den vier Fertigkeiten zu verbinden sind, könnte anhand des Lernfeldermodells gelöst werden. Inwiefern die Lehrenden damit bekannt sind, bedarf weiterer Untersuchung; angesichts der Forschungsergebnisse folgen viele wenigstens zum Teil - intuitiv? - bereits seinen Ideen. Nun sollten dafür allerdings auch andere im Rahmen von Seminaren und Fortbildungen entsprechend sensibilisiert werden.

\section{LITERATUR}

DRUMM, Sandra/Britta HUFEISEN/Johanna KLIPPEL (2013) Wie lernt man eigentlich Fremdsprachen? Sandra Ballweg/Sandra Drumm/Britta Hufeisen/ Johanna Klippel/Lina Pilypaityté, Deutsch lehren lernen 2: Wie lernt man die Fremdsprache Deutsch? München: Goethe-Institut, Klett-Langenscheidt, 14-64.

EUROPARAT (Hrsg.) (2001) Gemeinsamer Europäischer Referenzrahmen für Sprachen: lernen, lehren, beurteilen. 18. Februar 2018. https://www.goethe.de/Z/50/ commeuro/i0.htm.

FUNK, Hermann/Christina KUHN/Dirk SKIBA/Dorothea SPANIEL-WEISE/Rainer E. WICKE (2014) Deutsch lehren lernen 4: Aufgaben, Übungen, Interaktion. München: Goethe-Institut, Klett-Langenscheidt.

GLIHA OLENIK, Nadja/Vasja JAKŠE/Brigita KOSEVSKI PULJIĆ/Marinka KRENKER/Mojca LESKOVEC/Diana REDL KOLAR/Andreja RETELJ/Sonja ZVER (2018) Nemščina: predmetni izpitni katalog za splošno maturo. Ljubljana: Državni izpitni center. 27. Dezember 2020. https://www.ric.si/mma/MNEM-2020/2018083013280560/.

GNUTZMANN, Claus (2016) Sprachenbewusstheit und Sprachlernkompetenz. E. Burwitz-Melzer/G. Mehlhorn/C. Riemer/K.-R. Bausch/H.-J. Krumm (Hrsg.), Handbuch 
Fremdsprachenunterricht. 6., völlig überarbeitete und erweiterte Auflage. Tübingen: A. Francke Verlag, 144-149.

GOETHE-INSTITUT (2013) Didaktisch-methodische Prinzipien. 23. Februar 2018. https://www.goethe.de/resources/files/pdf157/unterrichtsprinzipien12.pdf.

GROTJAHN, Rüdiger/Karin KLEPPIN (2013) Handlungsorientierung, Kompetenzorientierung und weitere Prinzipien. K. Ende/R. Grotjahn/K. Kleppin/I. Mohr, Deutsch lehren lernen 6: Curriculare Vorgaben und Unterrichtsplanung. München: GoetheInstitut, Langenscheidt, 26-32.

HOLC, Nada/Stanka EMERŠIČ/Liljana KAČ/Ana Marija MUSTER/Herta OREŠIČ/ Nuša RUSTJA (2008) Učni načrt. Nemščina: gimnazija: splošna, klasična, strokovna gimnazija. Ljubljana: Ministrstvo za šolstvo in šport, Zavod RS za šolstvo. 13. August 2018. http://eportal.mss.edus.si/msswww/programi2010/programi/media/ pdf/un_gimnazija/un_nemscina_gimn.pdf.

KAČ, Liljana/Susanne VOLČANŠEK/Suzana RAMŠAK (2016) Program osnovna šola: Nemščina: Učni načrt. Ljubljana: Ministrstvo za izobraževanje, znanost in šport, Zavod RS za šolstvo. 6. August 2021. https://www.gov.si/assets/ministrstva/MIZS/ Dokumenti/Osnovna-sola/Ucni-nacrti/obvezni/UN_nemscina.pdf.

KLIPPEL, Friederike (2016) Didaktische und methodische Prinzipien der Vermittlung. E. Burwitz-Melzer/G. Mehlhorn/C. Riemer/K.-R. Bausch/H.-J. Krumm (Hrsg.), Handbuch Fremdsprachenunterricht. 6., völlig überarbeitete und erweiterte Auflage. Tübingen: A. Francke Verlag, 315-320.

KONDRIČ HORVAT, Vesna/Nada HOLC/Mihael KOLTAK/Tamara BOSNIČ/Brigita KOSEVSKI/Anica PLAZAR/Natalija ROBNIK (2001) Učni načrt za izbirni predmet: Tuji jezik nemščina. Ljubljana: Ministrstvo za šolstvo, znanost in šport, Zavod RS za šolstvo. 6. August 2021. https://www.gov.si/assets/ministrstva/MIZS/Dokumenti/Osnovna-sola/Ucni-nacrti/izbirni/3-letni/Nemscina_izbirni.pdf.

PEVEC SEMEC, Katica/Liljana KAČ/Neva ŠEČEROV/Simona CAJHEN/Barbara LESNIČAR/Alja LIPAVIC OŠTIR/Liliane STRMČNIK (2013) Program osnovna šola: Drugi tuji jezikv 4. do 9. razredu: Neobvezni izbirni predmet: Učni načrt. Ljubljana: Ministrstvo za izobraževanje, znanost in šport, Zavod Republike Slovenije za šolstvo. 6. August 2021. https://www.gov.si/assets/ministrstva/MIZS/Dokumenti/Osnovna-sola/Ucni-nacrti/izbirni/Neobvezni/Drugi_TJ_izbirni_neobvezni.pdf. 
POVZETEK

\section{ODNOS UČITELJEV IN UČITELJIC NEMŠČINE DO SLOVNICE PRI POUKU NEMŠČINE KOT TUJEGA JEZIKA NA PRIMERU ZUNANJIH OCENJEVALCEV IN OCENJEVALK SLOVENSKE MATURE}

V zgodovini didaktike tujih jezikov se je slovnici kot delni zmožnosti pripisoval različen pomen. Danes se kot najpomembnejši cilj pouka tujih jezikov v splošnem razume sporazumevalna zmožnost, Skupni evropski jezikovni okvir za jezike (2001) pa slovnično zmožnost opredeljuje kot njen osrednji sestavni del. Kljub temu pri sodobnem akcijsko usmerjenem in k učinkovitosti naravnanem pouku nemščine kot tujega jezika vloga slovnice ostaja sporna. Ta raziskava se je zato osredotočila na vprašanja, kakšno vlogo igra slovnica pri pouku nemščine kot tujega jezika v Sloveniji, kako se pri njem posredujejo slovnične strukture ter po katerih smernicah se učitelji in učiteljice pri tem ravnajo. Anketa je pokazala, da večina sodelujočih zunanjih ocenjevalcev in ocenjevalk slovenskega maturitetnega izpita iz nemščine, kot tudi drugih sodelujočih učiteljev in učiteljic nemščine kot tujega jezika v Sloveniji znanje slovnice opredeljuje kot pomembno za doseganje sporazumevalne zmožnosti v nemščini kot tujem jeziku in da ima slovnica pri njihovem pouku enakovredno vlogo kot ostale (delne) zmožnosti. Slovnične strukture sodelujoči učitelji in učiteljice posredujejo tako s postopki avtomatizacije kot s kognitivnimi postopki, razlago pri tem podajajo v kombinaciji prvega in ciljnega jezika, ravnajo pa se predvsem po lastnih izkušnjah in spoznanjih ter uporabljanih učbenikih. Mnogi sodelujoči učitelji in učiteljice torej vsaj delno že sledijo modelu štirih učnih področij, ki drugače kot tradicionalni model štirih jezikovnih zmožnosti slovnico integrira $v$ jezikovna opravila ter tako ločeni sestavini slovnico in besedišče povezuje s tradicionalnimi štirimi zmožnostmi.

Ključne besede: nemščina kot tuji jezik, sporazumevalna zmožnost, slovnična zmožnost, didaktično-metodična načela, akcijska usmerjenost

\section{ABSTRACT}

\section{ATTITUDES OF GFL TEACHERS TO GRAMMAR IN GFL CLASSROOMS USING THE EXAMPLE OF SLOVENIAN MATURA EXAMINERS}

In the history of foreign language teaching methodology, grammar, as a sub-skill, has been treated in very different ways. Today, the main goal to be achieved in foreign language teaching is generally understood to be communicative competence, and the Common European Framework of Reference for Languages (2001) describes grammatical competence as one of its central components. Nevertheless, the role of grammar remains contested in modern, efficiency- and action-oriented classrooms of German as a foreign language (GFL). The present research has 
therefore addressed the questions of the role that grammar plays in GFL classrooms in Slovenian schools, how the grammatical structures are presented and what is the orientation in this regard. A survey has shown that the majority of the participating matura examiners as well as the majority of other participating Slovenian teachers consider grammatical knowledge to be important for achieving communicative competence in GFL, and that grammar plays an equal role in their teaching as the other sub-skills. They use both drill-based and awareness-raising techniques to foster the acquisition of grammatical structures, explanations are given in a combination of the first and second languages, the teachers mainly orientate themselves based on their own experiences and knowledge, and the textbooks they are using. Many of them already follow the four strands model, which, in contrast to the traditional model of the four skills, integrates grammar into language activities and thus combines the separate components of grammar and vocabulary with the four skills.

Keywords: German as a foreign language, communicative competence, grammatical competence, teaching principles, action-oriented approach

\section{ZUSAMMENFASSUNG}

\section{DIE EINSTELLUNG DER DAF-LEHRENDEN ZUR GRAMMATIK IM DAF-UNTER- RICHT AM BEISPIEL SLOWENISCHER ABITURPRÜFENDER}

In der Geschichte der Fremdsprachendidaktik wurde der Grammatik als Teilkompetenz ein äußerst unterschiedlicher Stellenwert zugeschrieben. Als das wichtigste im Fremdsprachenunterricht zu erreichende Lernziel wird heute im Allgemeinen die kommunikative Kompetenz verstanden und der Gemeinsame europäische Referenzrahmen für Sprachen (2001) bezeichnet die grammatische Kompetenz als zentralen Bestandteil der kommunikativen Kompetenz. Dennoch bleibt im modernen effizienz- und handlungsorientierten Deutsch-als-Fremdsprache-Unterricht die Rolle der Grammatik umstritten. In der vorliegenden Untersuchung wurde folglich auf die Fragen eingegangen, was für eine Rolle Grammatik im DaF-Unterricht in Slowenien spielt, wie grammatische Strukturen vermittelt werden und was dabei die Orientierung darstellt. Eine Befragung hat gezeigt, dass die Mehrheit der teilnehmenden Abiturprüfenden sowie anderer teilnehmenden slowenischen Lehrenden Grammatikkenntnisse als wichtig für die Erreichung der kommunikativen Kompetenz im Deutschen als Zielsprache einschätzt und die Grammatik in ihrem Unterricht eine gleichwertige Rolle wie die anderen (Teil-)Kompetenzen spielt. Grammatische Strukturen vermitteln die Teilnehmenden sowohl mit automatisierenden als auch kognitivierenden Verfahren, die Erklärung erfolgt in einer Kombination der Erst- und Zielsprache, die Lehrenden orientieren sich dabei hauptsächlich an eigenen Erfahrungen und Erkenntnissen sowie an benutzten Lehrwerken. Viele teilnehmende Lehrende folgen zum Teil also bereits dem Lernfeldermodell, das anders als das traditionelle Modell der vier Fertigkeiten die Grammatik 
in das sprachliche Handeln integriert und somit die separaten Komponenten Grammatik und Wortschatz mit den vier Fertigkeiten verbindet.

Schlüsselwörter: Deutsch als Fremdsprache, kommunikative Kompetenz, grammatische Kompetenz, didaktisch-methodische Prinzipien, Handlungsorientierung 